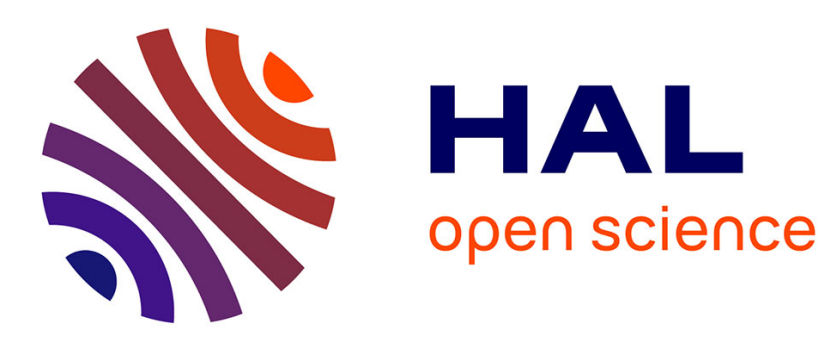

\title{
Few-optical-cycle dissipative solitons
}

Hervé Leblond, Dumitru Mihalache

\section{To cite this version:}

Hervé Leblond, Dumitru Mihalache. Few-optical-cycle dissipative solitons. Journal of Physics A: Mathematical and Theoretical, 2010, 43 (37), pp.375205. 10.1088/1751-8113/43/37/375205 . hal03187674

\section{HAL Id: hal-03187674 https://univ-angers.hal.science/hal-03187674}

Submitted on 1 Apr 2021

HAL is a multi-disciplinary open access archive for the deposit and dissemination of scientific research documents, whether they are published or not. The documents may come from teaching and research institutions in France or abroad, or from public or private research centers.
L'archive ouverte pluridisciplinaire HAL, est destinée au dépôt et à la diffusion de documents scientifiques de niveau recherche, publiés ou non, émanant des établissements d'enseignement et de recherche français ou étrangers, des laboratoires publics ou privés. 
Few-optical-cycle dissipative solitons

This content has been downloaded from IOPscience. Please scroll down to see the full text.

2010 J. Phys. A: Math. Theor. 43375205

(http://iopscience.iop.org/1751-8121/43/37/375205)

View the table of contents for this issue, or go to the journal homepage for more

Download details:

IP Address: 193.52.40.1

This content was downloaded on 24/02/2015 at 12:05

Please note that terms and conditions apply. 


\title{
Few-optical-cycle dissipative solitons
}

\author{
H Leblond $^{1}$ and D Mihalache ${ }^{2,3}$ \\ ${ }^{1}$ Laboratoire de Photonique d'Angers EA 4464, Université d'Angers, 2 Bd. Lavoisier, \\ 49045 Angers Cedex 01, France \\ ${ }^{2}$ Horia Hulubei National Institute for Physics and Nuclear Engineering (IFIN-HH), 407 \\ Atomistilor, Magurele-Bucharest, 077125, Romania \\ ${ }^{3}$ Academy of Romanian Scientists, 54 Splaiul Independentei, Bucharest 050094, Romania \\ E-mail: herve.leblond@univ-angers.fr
}

Received 2 February 2010, in final form 25 June 2010

Published 2 August 2010

Online at stacks.iop.org/JPhysA/43/375205

\begin{abstract}
By using a powerful reductive perturbation technique, or multiscale analysis, a generalized modified Korteweg-de Vries partial differential equation is derived, which describes the physics of few-optical-cycle dissipative solitons beyond the slowly varying envelope approximation. Numerical simulations of the formation of stable dissipative solitons from arbitrary breather-like few-cycle pulses are also given.
\end{abstract}

PACS numbers: $42.65 . \mathrm{Tg}$, 42.65.Re, 05.45.Yv

(Some figures in this article are in colour only in the electronic version)

\section{Introduction}

Ultrafast nonlinear optics has led to the production, measurement and control of pulses with duration down to a few optical cycles, see [1]. The study of these intense optical pulses has opened the door to a bunch of applications in various fields such as light matter interaction, high-order harmonic generation, extreme nonlinear optics [2] and attosecond physics, which deals with both measurement and control of the subcycle field evolution of few-cycle light pulses $[3,4]$. The theoretical studies of the physics of few-cycle pulses (FCPs) concentrated on three classes of governing dynamical models: (i) the quantum approach [5-8], (ii) the refinements within the framework of the slowly varying envelope approximation (SVEA) of the nonlinear Schrödinger-type envelope equations [9-11] and (iii) non-SVEA models [12-16]. In media with cubic optical nonlinearity (Kerr media) the physics of FCPs can be adequately described beyond the SVEA by using different models, such as the modified Korteweg-de Vries (mKdV) [12], sine-Gordon (sG) [13, 14] or $\mathrm{mKdV-sG} \mathrm{equations}[15,16]$. It is worthy to mention that the $\mathrm{mKdV}$ and $\mathrm{sG}$ equations are completely integrable by means of the inverse scattering transform method, see, for example, [17, 18], whereas the $\mathrm{mKdV-sG}$ equation is completely integrable only if a certain condition between its coefficients is fulfilled [19]. 
The above-mentioned evolution equations admit breather solutions, which are suitable for describing the physics of few-optical-cycle solitons. Moreover, a non-integrable generalized Kadomtsev-Petviashvili equation (a two-dimensional version of the mKdV model) was also put forward for describing the two-dimensional few-optical-cycle soliton propagation [20, 21].

Of particular interest for the physics of FCPs is the mKdV-sG equation; thus, by using a system of two-level atoms, it has been shown in [15] that the propagation of ultrashort pulses in Kerr optical media is fairly well described by a $\mathrm{mKdV}$-sG equation. It is worthy to mention that this quite general model was also derived and studied in $[22,23]$. However, another model equation for describing the physics of FCPs known as the short-pulse equation (SPE) has been introduced, too [24-27], and some vectorial versions of the SPE have also been investigated [28-30]. Another kind of SPE containing an additional dispersion term has been introduced in [31]. A multi-dimensional version of the SPE was also put forward [32] and self-focusing and pulse compression have been studied, too [33]. The SPE model has been considered again in its vectorial version, and the pulse self-compression and FCP soliton propagation have been investigated in detail [34]. Recently, we have revisited the main dynamical models mentioned above, which describe the $(1+1)$-dimensional propagation of few-optical-cycle pulses in transparent media [35]. We have thus proved that the dynamical model based on the $\mathrm{mKdV}$-sG partial differential equation was able to retrieve the results reported so far in the literature, and so demonstrating its remarkable mathematical capabilities in describing the physics of FCP optical solitons [35].

However, there are only a few works devoted to the study of few-optical-cycle dissipative solitons. It is worthy to mention the works of Rosanov et al [6, 7], where both the formation of few-optical-cycle dissipative solitons in active nonlinear optical fibres and the collisions between them were investigated. The theory of mode-locked lasers essentially relies on mean field models derived within the SVEA. One of the most important of such models is Haus' master equation [36], which is in fact the stationary version of the complex Ginzburg-Landau (CGL) equation (see [37, 38]). The short pulses are fairly well described by soliton solutions to the CGL equation, which are unstable for the cubic CGL and stable for the cubic-quintic one. Both the cubic and the quintic CGL models have been derived from a detailed description of the laser cavity, in the case of fibre lasers mode locked by means of nonlinear polarization rotation or figure 8 ones [39-41]. Note that, for such lasers, descriptions of the cavity by means of full numerical resolution of propagation equations along it also have been used, see e.g. [42]. The Lorentz-Haken equations, commonly considered as a general model of a laser setup, have been reduced to the Swift-Hohenberg equation [43], which can be considered as a perturbed CGL equation [37]. It is commonly admitted that mode locking requires some saturable absorber [44] or some setup having an equivalent effect; nonlinear polarization rotation and nonlinear loop mirror have been mentioned above. An alternative technique is the Kerr lens mode locking (KLM) [45-47], which combines self-focusing due to the Kerr effect and the use of an aperture to select the highest intensities. KLM is not a mere effective amplitudedependent gain/loss effect, but a complex phenomenon which involves the spatiotemporal intra-cavity pulse dynamics. The existing theory of KLM is based on nonlinear geometrical optics for Gaussian beams, hence within the SVEA, and the initial approach of [45-47] was not fundamentally modified in more recent studies (see e.g. [48, 49]). The technique is very efficient even in the case of strong self-focusing and two-cycle pulses (see e.g. [50]), in which the validity of the approximations used in the theoretical developments is not ensured.

The aim of this paper is to derive a generic partial differential equation describing the dynamics of dissipative FCP solitons in a laser cavity filled with two-level atoms, beyond SVEA model equations. Starting from the Maxwell-Bloch equations, by using a multiscale perturbation approach [51] we derive a non-SVEA version of the Lorenz-Haken equations 
$[52,53]$. We have obtained a generic equation describing dissipative few-optical-cycle solitons in the form of a generalized $\mathrm{mKdV}$ equation containing additional terms accounting for gain and losses. Thus in addition to the standard term accounting for linear losses (proportional to the optical field) we get a term proportional to the second-order derivative of the optical field with respect to the time variable, which accounts for gain and a second, regularizing term proportional to the fourth-order derivative with respect to the time variable of the optical field, which accounts for losses.

This paper is organized as follows. In section 2 we introduce a model based on the Maxwell-Bloch equation for an ensemble of two-level atoms, which takes into account both the losses due to the optical field reflection at the laser cavity boundaries and the ones occurring in the medium itself, by means of two additional atomic levels. In section 3 we evaluate the mirror losses and we get the corresponding modified Maxwell-Bloch equations. Section 4 is devoted to the multiscale approach up to the third order in a certain small perturbation parameter $\varepsilon$ so that the orders of magnitude of the various physical effects are determined by means of their order in an expansion in a series of powers of $\varepsilon$. The outcome of this reductive perturbation method (or multiscale analysis) is a generalized $\mathrm{mKdV}$ equation containing gain and loss terms. In section 5 we discuss the necessity of introducing an additional, regularizing term in the generalized $\mathrm{mKdV}$ equation obtained in section 4 , which accounts for losses in the system. Section 6 is devoted to the numerical resolution of the generalized mKdV equation which fairly well describes the physics of few-optical-cycle dissipative solitons. Finally, section 7 concludes the paper.

\section{Maxwell-Bloch equations}

More than three decades ago, Haken [53] used a single-mode unidirectional ring laser model (with a homogeneously broadened line) described by the Maxwell-Bloch equations and after some approximations showed its mathematical equivalence with an appropriate model of the Lorenz oscillator [52]. The aim of this work is to derive a non-SVEA version of the Lorenz-Haken equations. We start from the Maxwell-Bloch equations

$$
\partial_{z}^{2} \mathbf{E}=\frac{1}{c^{2}} \partial_{t}^{2}(\mathbf{E}+\hat{\mathbf{P}})
$$

with

$$
\hat{\mathbf{P}}=N \operatorname{Tr}(\hat{\rho} \hat{\mu}) .
$$

Here $N$ is the atomic density, $\hat{\mu}$ is the dipolar momentum operator and $\hat{\rho}$ is the density matrix, which obeys the Schrödinger equation

$$
\mathrm{i} \hbar \partial_{t} \hat{\rho}=\left[\hat{H}_{0}-\hat{\mu} \cdot \hat{\mathbf{E}}, \hat{\rho}\right]+\mathrm{i} \hat{\Lambda}-\mathrm{i} \hat{R},
$$

where $[\cdot, \cdot]$ holds for the commutator operation, $\hat{\Lambda}$ accounts for gain and $\hat{R}$ for relaxation of the components of the density matrix $\hat{\rho}$. In the following we restrict ourselves to the case of linear polarization, that is, we consider scalar fields only; thus, we next write $E, \hat{P}, \hat{\mu}$ instead of $\mathbf{E}, \hat{\mathbf{P}}, \hat{\mu}$. In equation (3) $\hat{H}_{0}$ is the free Hamiltonian of the atomic system under consideration. However, losses must also be taken into account. A phenomenological loss term has to be introduced in such a way that it can be compensated by gain. It is necessary that the time evolution of losses (or their spectral profile) be able to adjust to the gain. Hence not only losses due to reflection at the cavity boundaries, but also the ones of the medium must be taken into account. Therefore we need to introduce a second transition, independent of the 
former (pumped) one, which only produces absorption. The corresponding free Hamiltonian is thus

$$
\hat{H}_{0}=\left(\begin{array}{cc}
H_{0} & 0 \\
0 & H_{0}^{\prime}
\end{array}\right)=\hbar\left(\begin{array}{cccc}
\omega_{a} & 0 & 0 & 0 \\
0 & \omega_{b} & 0 & 0 \\
0 & 0 & \omega_{a}^{\prime} & 0 \\
0 & 0 & 0 & \omega_{b}^{\prime}
\end{array}\right)
$$

Here, as we said before, we consider a set of four-level atoms with the Hamiltonian $\hat{H}_{0}$, corresponding to two distinct two-level transitions with frequencies $\Omega=\omega_{b}-\omega_{a}$ and $\Omega^{\prime}=\omega_{b}^{\prime}-\omega_{a}^{\prime}$, respectively. The dipolar momentum is

$$
\hat{\mu}=\left(\begin{array}{cc}
\mu & 0 \\
0 & \mu^{\prime}
\end{array}\right)=\left(\begin{array}{cccc}
0 & \mu & 0 & 0 \\
\mu^{*} & 0 & 0 & 0 \\
0 & 0 & 0 & \mu^{\prime} \\
0 & 0 & \mu^{\prime *} & 0
\end{array}\right),
$$

the gain is

$$
\hat{\Lambda}=\left(\begin{array}{ll}
\Lambda & 0 \\
0 & 0
\end{array}\right)=\left(\begin{array}{cccc}
\lambda_{a} & 0 & 0 & 0 \\
0 & \lambda_{b} & 0 & 0 \\
0 & 0 & 0 & 0 \\
0 & 0 & 0 & 0
\end{array}\right)
$$

and the relaxation term is

$$
\hat{R}=\left(\begin{array}{cc}
R & 0 \\
0 & R^{\prime}
\end{array}\right)=\left(\begin{array}{cccc}
\gamma_{a}\left(\rho_{a}-\alpha\right) & \gamma_{t} \rho_{t} & 0 & 0 \\
\gamma_{t} \rho_{t}^{*} & \gamma_{a} \rho_{b} & 0 & 0 \\
0 & 0 & \gamma_{a}^{\prime}\left(\rho_{a}^{\prime}-\alpha\right) & \gamma_{t} \rho_{t}^{\prime} \\
0 & 0 & \gamma_{t}^{\prime} \rho_{t}^{\prime *} & \gamma_{a}^{\prime} \rho_{b}^{\prime}
\end{array}\right)
$$

with $\alpha=1$ in standard normalization, and

$$
\hat{\rho}=\left(\begin{array}{cccc}
\rho_{a} & \rho_{t} & \rho_{13} & \rho_{14} \\
\rho_{t}^{*} & \rho_{b} & \rho_{23} & \rho_{24} \\
\rho_{31} & \rho_{32} & \rho_{a}^{\prime} & \rho_{t}^{\prime} \\
\rho_{41} & \rho_{42} & \rho_{t}^{\prime *} & \rho_{b}^{\prime}
\end{array}\right) .
$$

Next it can be easily shown that the matrix $\hat{\rho}$ is diagonal:

$$
\hat{\rho}=\left(\begin{array}{cc}
\rho & 0 \\
0 & \rho^{\prime}
\end{array}\right),
$$

i.e. $\rho_{13}=\rho_{14}=\rho_{23}=\rho_{24}=\rho_{31}=\rho_{32}=\rho_{41}=\rho_{42}=0$. Thus the Schrödinger equation (3) splits into two equations of the same form for the two diagonal blocks. Each of these equations describes a two-level transition.

\section{Evaluation of mirror losses}

In order to derive a differential term which can account for the losses due to the mirrors, we consider a strongly simplified cavity model, in which only the mirrors are taken into account. Consider thus a cavity with length $L$, and mirrors with amplitude reflection coefficients $r$. We will describe it as a periodic medium with period $L$ and periodically localized losses with a 
loss factor $\left(1-r^{2}\right)$. Let $z$ be the variable along the cavity axis and $v$ the light velocity. During a given round trip, between the two mirrors, the electric field is expressed as

$$
E(z, t)=E_{+}(z-v t)+E_{-}(z+v t)
$$

Let us now consider the wave $E_{+}$, which propagates towards positive $z$ during the considered round trip. For large times, its expression becomes complicated. More precisely, after the propagation time $T=n L / v$, the wave has covered a distance $Z=n L$, and the wave has crossed the mirror $n$ times; hence,

$$
E_{+}(z+Z, t+T)=r^{n} E_{+}(z-v t)=\mathrm{e}^{(v T \ln r) / L} E_{+}(z-v t) .
$$

For large $n$, a continuous approximation is performed, assuming that (11) holds for any real $T$. A change of variables then yields for large enough $t^{\prime}$

$$
E_{+}\left(z^{\prime}, t^{\prime}\right)=E_{+}\left(z^{\prime}-v t^{\prime}\right) \mathrm{e}^{-\beta\left(t^{\prime}-t_{0}\right)},
$$

where we have the set $\beta=-(v \ln r) / L$. We find in the same way

$$
E_{-}\left(z^{\prime}, t^{\prime}\right)=E_{-}\left(z^{\prime}+v t^{\prime}\right) \mathrm{e}^{-\beta\left(t^{\prime}-t_{0}\right)} .
$$

Dropping the primes and setting $t_{0}=0$ without loss of generality, and within the above continuous approximation for large $t$, which in nothing else but the mean field approximation, we get

$$
E(z, t)=E_{+}(z-v t) \mathrm{e}^{-\beta t}+E_{-}(z+v t) \mathrm{e}^{-\beta t} .
$$

Then taking twice the $t$-derivative of (14), and making use of the relation

$$
v\left(-\partial_{z} E_{+}+\partial_{z} E_{-}\right) \mathrm{e}^{-\beta t}=\partial_{t} E+\beta E
$$

we find that $E$ satisfies the differential equation

$$
\partial_{t}^{2} E=v^{2} \partial_{z}^{2} E-2 \beta \partial_{t} E-\beta^{2} E,
$$

or

$$
\partial_{z}^{2} E=\frac{1}{v^{2}}\left(\beta+\partial_{t}\right)^{2} E
$$

Equation (17) indicates us how the Maxwell-Bloch wave equation (1) should be modified in order to take into account the mirror losses. Equation (1) is thus replaced with

$$
\partial_{z}^{2} E=\frac{1}{c^{2}}\left(\beta+\partial_{t}\right)^{2}\left(E+P+P^{\prime}\right)
$$

where $\beta$ is the mirror loss parameter and $P$ and $P^{\prime}$ are the two polarization terms corresponding to the two distinct transitions, see section 2 .

\section{Multiscale approach}

It is well known that the reductive perturbation method is a very powerful way of deriving simplified, generic models describing nonlinear wave propagation and interaction in various physics settings [51]. We next introduce scaled variables corresponding to a long-wave approximation of $\mathrm{mKdV}$ type, as

$$
\begin{aligned}
\tau & =\varepsilon\left[t-z\left(\frac{1}{V}+\varepsilon W\right)\right], \\
\zeta & =\varepsilon^{3} z .
\end{aligned}
$$


With respect to the standard mKdV-type scaling, expression (19) involves an additional correction of order $\varepsilon$ to the speed. We expand the optical field $E$ in a power series of a small parameter $\varepsilon$ as

$$
E=\varepsilon E_{1}(\zeta, \tau)+\varepsilon^{2} E_{2}(\zeta, \tau)+\cdots
$$

The corresponding power series expansions of $\rho$ and $\rho^{\prime}$ start with the order $\varepsilon^{0}$ quantity $\rho_{0}$. The gain $\Lambda$, and $R$ are also expanded in this way. In [13] was assumed a weak damping, in order to account for propagation in a transparent medium. No approximation of this kind is relevant here. We indeed consider a laser setup; hence, the characteristic frequency $\omega_{0}$ of the wave must be close to the resonance line, say $\Omega=\omega_{b}-\omega_{a}$, and especially fall within the gain curve. Since we use a long wave approximation, we have (at least formally) $\omega_{0} \simeq 0$. Hence the spectral line, which is expressed typically as

$$
\frac{1}{(\omega-\Omega)^{2}+\gamma_{t}^{2}}
$$

must extend down to $\omega=\omega_{0} \simeq 0$, which happens only if its width $\gamma_{t}$ has the same order of magnitude as the central line $\Omega$. The second transition, with central line $\Omega^{\prime}$, has been introduced to account for cavity losses. Further, losses must be compensated by gain. Therefore it is necessary that the bandwidths of both gain and losses have the same order of magnitude. Thus $\gamma_{t}^{\prime}$ must have the same order of magnitude as $\gamma_{t}$.

In order to give the physical interpretation of the present scaling, let us consider the inverse of the spectral width $\gamma_{t}$ as a reference frequency of order $\varepsilon^{0}$. The long-wave approximation means that the characteristic frequency $\omega_{0}$ of the wave is small with respect to $\gamma_{t}$, or conversely, that $\gamma_{t}$ is large with respect to $\omega_{0}$. The long-wave approximation can thus be seen as an approximation of large bandwidth. It is indeed well known that a very large bandwidth is required to produce ultrashort pulses.

On the other hand, the mirror loss parameter $\beta$ is expanded as $\beta=\varepsilon \beta_{1}+\varepsilon^{2} \beta_{2}+\cdots$. Hence we assume that $\beta$ is small (good cavity condition). However, the order of magnitude of $\beta$ which allows dissipative soliton propagation is not specified $a$ priori; it will arise as a result of the computation, see below.

We use the same normalization as in [13]. Thus setting

$$
\begin{aligned}
& \tilde{t}=c t, \quad \tilde{P}=4 \pi P, \quad \tilde{\rho}=4 \pi N \hbar c \rho, \quad \tilde{\mu}=\frac{\mu}{\hbar c}, \\
& \tilde{H}_{0}=\frac{H_{0}}{\hbar c}, \quad \tilde{\omega}_{a, b}=\frac{\omega_{a, b}}{c},
\end{aligned}
$$

and analogously for other operators and variables with the same physical dimension, allows us to replace the constants $c, N, \hbar$ and $4 \pi$ in equations (1), (3) by 1 . In what follows we drop the tilde. Note that, according to the change of variables (23), the trace $\operatorname{Tr}(\rho)$ of the density matrix is not 1 but $\alpha=4 \pi N \hbar c$. Now let us proceed with the order-by-order resolution of the governing equations (1)-(3).

\subsection{Order 0}

The Schrödinger equation for the first transition (let us call it equation $(S)$ ), at order $\varepsilon^{0}$, is

$$
0=\left[H_{0}, \rho_{0}\right]+\mathrm{i} \Lambda_{0}-\mathrm{i} R_{0} .
$$

Using

$$
\left[H_{0}, \rho_{0}\right]=\left(\begin{array}{cc}
0 & -\Omega \rho_{t} \\
\Omega \rho_{t}^{*} & 0
\end{array}\right)
$$

6 
we get $\rho_{0 t}=0$, and

$$
\rho_{0 a}=\alpha+\frac{\lambda_{0 a}}{\gamma_{a}}, \quad \rho_{0 b}=\frac{\lambda_{0 b}}{\gamma_{a}} .
$$

Due to the condition $\operatorname{Tr} \rho=\alpha$, we see that $\lambda_{0 b}=-\lambda_{0 a}$. We define the population inversion as $w_{j}=\rho_{j b}-\rho_{j a}$ for all $j$; then

$$
w_{0}=\frac{2 \lambda_{0 b}}{\gamma_{a}}-\alpha
$$

We will assume that $w_{0}>0$; hence $\lambda_{0 b}>\alpha \gamma_{a} / 2$ and especially $\lambda_{0 b}>0$.

For the Schrödinger equation for the second transition, which we call equation $\left(S^{\prime}\right)$, we get the same results, but without pumping, and with the corresponding 'prime' quantities, i.e.

$$
\begin{aligned}
& \rho_{0 a}^{\prime}=\alpha, \quad \rho_{0 b}^{\prime}=0, \quad \rho_{0 t}^{\prime}=0, \\
& w_{0}^{\prime}=\rho_{0 b}^{\prime}-\rho_{0 a}^{\prime}=-\alpha<0 .
\end{aligned}
$$

\subsection{Order 1}

Equation $(S)$ at order $\varepsilon$ is

$$
\mathrm{i} \partial_{\tau} \rho_{0}=\left[H_{0}, \rho_{1}\right]-E_{1}\left[\mu, \rho_{0}\right]+\mathrm{i} \Lambda_{1}-\mathrm{i} R_{1} .
$$

Making use of

$$
\left[\mu, \rho_{j}\right]=\left(\begin{array}{cc}
\mu \rho_{j t}^{*}-\mu^{*} \rho_{j t} & \mu w_{j} \\
-\mu^{*} w_{j} & \mu^{*} \rho_{j t}-\mu \rho_{j t}^{*}
\end{array}\right),
$$

and taking into account that $\rho_{0 t}=0$, we get

$$
\begin{aligned}
& w_{1}=-2 \rho_{1 a}=2 \rho_{1 b}=\frac{2 \lambda_{1 b}}{\gamma_{a}}, \\
& \rho_{1 t}=\frac{-\mu w_{0} E_{1}}{\Omega+i \gamma_{t}} .
\end{aligned}
$$

The polarization term is deduced from equation (2), which is written explicitly as

$$
P_{j}=\mu^{*} \rho_{j t}+\text { c.c., }
$$

where c.c. stands for complex conjugate. Hence

$$
P_{1}=\frac{-2 \Omega|\mu|^{2} w_{0} E_{1}}{\Omega^{2}+\gamma_{t}^{2}}
$$

For equation $\left(S^{\prime}\right)$, we get analogous formulae, with $\lambda_{1 b}$ replaced by 0 and $w_{0}^{\prime}=-\alpha$.

The wave equation (18) at leading order $\varepsilon^{3}$ is

$$
\frac{1}{V^{2}} \partial_{\tau}^{2} E_{1}=\left(\beta_{1}^{2}+2 \beta_{1} \partial_{\tau}+\partial_{\tau}^{2}\right)\left(E_{1}+P_{1}+P_{1}^{\prime}\right)
$$

Since $P_{1}, P_{1}^{\prime} \propto E_{1}$, equation (37) admits a nonzero stationary solution only if $\beta_{1}=0$ and

$$
\frac{1}{V^{2}}=1+\frac{2 \Omega^{\prime}\left|\mu^{\prime}\right|^{2} \alpha}{\Omega^{\prime 2}+\gamma_{t}^{\prime 2}}-\frac{2 \Omega|\mu|^{2} w_{0}}{\Omega^{2}+\gamma_{t}^{2}}
$$

It is worthy to mention that the velocity $V^{2}$ has the same expression as in [13], except that (i) there are two terms in the above equation, one term for each of the two transitions, (ii) the line widths $\gamma_{t}, \gamma_{t}^{\prime}$ are not neglected and (iii) $w_{0}>0$, which accounts for gain. 


\subsection{Order 2}

Equation $(S)$ at order $\varepsilon^{2}$ is

$$
\mathrm{i} \partial_{\tau} \rho_{1}=\left[H_{0}, \rho_{2}\right]-E_{2}\left[\mu, \rho_{0}\right]-E_{1}\left[\mu, \rho_{1}\right]+\mathrm{i} \Lambda_{2}-\mathrm{i} R_{2} .
$$

The $a$-component of equation (39) is

$$
0=-E_{1}\left(\mu \rho_{1 t}^{*}-\mu^{*} \rho_{1 t}\right)+\mathrm{i} \lambda_{2 a}-\mathrm{i} \gamma_{a} \rho_{2}^{a} .
$$

Then using (34) we get

$$
w_{2}=2 \rho_{2 b}=-2 \rho_{2 a}=\frac{2 \lambda_{2 b}}{\gamma_{a}}-\frac{4 \gamma_{t}|\mu|^{2} w_{0} E_{1}^{2}}{\gamma_{a}\left(\Omega^{2}+\gamma_{t}^{2}\right)} .
$$

The $t$-component of equation (39) is

$$
\mathrm{i} \partial_{\tau} \rho_{1 t}=-\Omega \rho_{2 t}-E_{2} \mu w_{0}-E_{1} \mu w_{1}-\mathrm{i} \gamma_{t} \rho_{2 t},
$$

and by using (34) it yields the expression of $\rho_{2 t}$ and hence the polarization is

$$
P_{2}=\frac{-2 \Omega|\mu|^{2}}{\Omega^{2}+\gamma_{t}^{2}}\left(E_{2} w_{0}+E_{1} w_{1}\right)+\frac{4 \Omega \gamma_{t}|\mu|^{2} w_{0}}{\left(\Omega^{2}+\gamma_{t}^{2}\right)^{2}} \partial_{\tau} E_{1} .
$$

For equation $\left(S^{\prime}\right)$, we get similar results, excepting that there is no pumping $\left(\lambda_{2 b}\right.$ is replaced by zero and $\left.w_{0}^{\prime}=-\alpha\right)$, and $w_{1}^{\prime}=0$.

Since $\beta_{1}=0$, the wave equation (18) at perturbation order $\varepsilon^{4}$ is

$$
\frac{1}{V^{2}} \partial_{\tau}^{2} E_{2}+\frac{2 W}{V} \partial_{\tau}^{2} E_{1}=\partial_{\tau}^{2}\left(E_{2}+P_{2}+P_{2}^{\prime}\right)+2 \beta_{2} \partial_{\tau}\left(E_{1}+P_{1}+P_{1}^{\prime}\right)
$$

Using equation (38), the terms involving $E_{2}$ vanish, and equation (44) reduces to

$$
\frac{2 W}{V} \partial_{\tau}^{2} E_{1}=\partial_{\tau}^{2}\left[\frac{-2 \Omega|\mu|^{2}}{\Omega^{2}+\gamma_{t}^{2}} E_{1} w_{1}+\frac{4 \Omega \gamma_{t}|\mu|^{2} w_{0}}{\left(\Omega^{2}+\gamma_{t}^{2}\right)^{2}} \partial_{\tau} E_{1}-\frac{4 \Omega^{\prime} \gamma_{t}^{\prime}\left|\mu^{\prime}\right|^{2} \alpha}{\left(\Omega^{\prime 2}+\gamma_{t}^{\prime 2}\right)^{2}} \partial_{\tau} E_{1}\right]+\frac{2 \beta_{2}}{V^{2}} \partial_{\tau} E_{1},
$$

for which we also used equation (43) and the expression of $P_{2}^{\prime}$. Equation (45) will not admit localized stationary solutions, except if the coefficients of $\partial_{\tau} E_{1}, \partial_{\tau}^{2} E_{1}$ and $\partial_{\tau}^{3} E_{1}$ are all zero. First, we get $\beta_{2}=0$, which implies that mirror losses are even smaller as previously estimated. Second, we obtain a relationship between $W$ and $w_{1}$

$$
W=\frac{-V \Omega|\mu|^{2}}{\Omega^{2}+\gamma_{t}^{2}} w_{1}
$$

i.e. a correction $w_{1}$ of order $\varepsilon$ to the population inversion induced a correction of the same order to the velocity. Third we are left with the relationship

$$
\frac{4 \Omega \gamma_{t}|\mu|^{2} w_{0}}{\left(\Omega^{2}+\gamma_{t}^{2}\right)^{2}}=\frac{4 \Omega^{\prime} \gamma_{t}^{\prime}\left|\mu^{\prime}\right|^{2} \alpha}{\left(\Omega^{\prime 2}+\gamma_{t}^{\prime 2}\right)^{2}}
$$

i.e. the gain induced by the pumped transition compensates the losses induced by the unpumped one, at this perturbation order. Equation (47) determines the laser threshold. An essential feature of laser pulse propagation is the excess of gain above the threshold. It is due to the correction term $w_{1}$ to the population inversion, and fixes the magnitude of the gain term in the evolution equation (73) obtained here governing the dynamics of few-optical-cycle dissipative solitons (see equation (55) below), as in SVEA models of Ginzburg-Landau type [54]. On the other hand, due to equation (38), the velocity $V$ depends on the population inversion, and hence a correction to the velocity arises as a consequence of the existence of the excess of gain $w_{1}$, according to equation (46). 


\subsection{Order 3}

Equation $(S)$ at order $\varepsilon^{3}$ is

$$
\mathrm{i} \partial_{\tau} \rho_{2}=\left[H_{0}, \rho_{3}\right]-E_{3}\left[\mu, \rho_{0}\right]-E_{2}\left[\mu, \rho_{1}\right]-E_{1}\left[\mu, \rho_{2}\right]+\mathrm{i} \Lambda_{3}-\mathrm{i} R_{3} .
$$

The $t$-component of equation (48) is written as

$$
\mathrm{i} \partial_{\tau} \rho_{2 t}=-\Omega \rho_{3 t}-E_{3} \mu w_{0}-E_{2} \mu w_{1}-E_{1} \mu w_{2}-\mathrm{i} \gamma_{t} \rho_{3 t} .
$$

Using the expression of $\rho_{2 t}$ and equation (41), we get $\rho_{3 t}$ and consequently the polarization is

$$
\begin{aligned}
P_{3}=\frac{-2 \Omega|\mu|^{2}}{\Omega^{2}+\gamma_{t}^{2}} & \left(E_{3} w_{0}+E_{2} w_{1}\right)-\frac{4 \lambda_{2 b} \Omega|\mu|^{2}}{\gamma_{a}\left(\Omega^{2}+\gamma_{t}^{2}\right)} E_{1}+\frac{8 \Omega \gamma_{t}|\mu|^{4} w_{0} E_{1}^{3}}{\gamma_{a}\left(\Omega^{2}+\gamma_{t}^{2}\right)^{2}} \\
+ & \frac{4 \Omega \gamma_{t}|\mu|^{2}}{\left(\Omega^{2}+\gamma_{t}^{2}\right)^{2}}\left(w_{0} \partial_{\tau} E_{2}+w_{1} \partial_{\tau} E_{1}\right)+\frac{2 \Omega\left(\Omega^{2}-3 \gamma_{t}^{2}\right)|\mu|^{2} w_{0}}{\left(\Omega^{2}+\gamma_{t}^{2}\right)^{3}} \partial_{\tau}^{2} E_{1} .
\end{aligned}
$$

For equation $\left(S^{\prime}\right)$ we get analogous expressions (with $w_{0}^{\prime}=-\alpha, w_{1}^{\prime}=0$, and $\lambda_{2 b}$ replaced with 0 , as above).

The wave equation (18) at perturbation order $\varepsilon^{5}$ is, since $\beta_{1}=\beta_{2}=0$, $\frac{1}{V^{2}} \partial_{\tau}^{2} E_{3}+\frac{2 W}{V} \partial_{\tau}^{2} E_{2}+W^{2} \partial_{\tau}^{2} E_{1}-\frac{2}{V} \partial_{\zeta} \partial_{\tau} E_{1}=\partial_{\tau}^{2}\left(E_{3}+P_{3}+P_{3}^{\prime}\right)+2 \beta_{3} \partial_{\tau}\left(E_{1}+P_{1}+P_{1}^{\prime}\right)$

The terms involving the field $E_{3}$ cancel from equation (51) due to condition (38). The terms involving the field $E_{2}$ cancel due to conditions (46) (i.e. due to the correction to speed) and (47) (i.e. the condition that gain compensates losses). It remains the following equation for $E_{1}$ :

with

$$
\partial_{\zeta} E_{1}=-A \partial_{\tau} E_{1}^{3}-B \partial_{\tau}^{3} E_{1}-C \partial_{\tau}^{2} E_{1}+D \partial_{\tau} E_{1}-\Gamma E_{1},
$$

$$
\begin{aligned}
& A=\frac{4 V \Omega \gamma_{t}|\mu|^{4} w_{0}}{\gamma_{a}\left(\Omega^{2}+\gamma_{t}^{2}\right)^{2}}-\frac{4 V \Omega^{\prime} \gamma_{t}^{\prime}\left|\mu^{\prime}\right|^{4} \alpha}{\gamma_{a}^{\prime}\left(\Omega^{\prime 2}+\gamma_{t}^{\prime 2}\right)^{2}} \\
& B=\frac{V \Omega\left(\Omega^{2}-3 \gamma_{t}^{2}\right)|\mu|^{2} w_{0}}{\left(\Omega^{2}+\gamma_{t}^{2}\right)^{3}}-\frac{V \Omega^{\prime}\left(\Omega^{\prime 2}-3 \gamma_{t}^{\prime 2}\right)\left|\mu^{\prime}\right|^{2} \alpha}{\left(\Omega^{\prime 2}+\gamma_{t}^{\prime 2}\right)^{3}}, \\
& C=\frac{2 V \Omega \gamma_{t}|\mu|^{2} w_{1}}{\left(\Omega^{2}+\gamma_{t}^{2}\right)^{2}} \\
& D=\frac{2 V \lambda_{2 b} \Omega|\mu|^{2}}{\gamma_{a}\left(\Omega^{2}+\gamma_{t}^{2}\right)}+\frac{V W^{2}}{2}
\end{aligned}
$$

and

$$
\Gamma=\frac{\beta_{3}}{V} .
$$

It is worthy to mention that for $C=D=\Gamma=0$, equation (52) reduces to the mKdV equation. The term containing the second-order derivative with respect to the time variable $\tau$ looks like a diffusion term in a partial differential equation of Burgers' type (see, for example, [17] and [18]). However, if we assume $\lambda_{1 b}=\gamma_{a} w_{1} / 2>0$, i.e. for a pumping slightly above the threshold characterized by equation (47), then $C>0$ and this term becomes a gain one. The term proportional to first-order derivative with respect to the time variable $\tau$ corresponds to a pumping term of lower order, but its concrete contribution here is a mere change in velocity. Thus the parameter $D$ can be changed to any value at our convenience by means of adequate Galilean transform. The term $\left(-\Gamma E_{1}\right)$ corresponds to the losses due to the mirrors, with $\beta_{3}>0$ and $\Gamma>0$. 


\section{An additional regularizing term}

The trouble is that equation (52) is highly unstable when $C$ is positive. Indeed, if we make a Fourier transform of the field

$$
E_{1}=\int \hat{E}(\omega) \mathrm{e}^{\mathrm{i} \omega t} \mathrm{~d} \omega
$$

then the Fourier transform of the gain term is

$$
\mathcal{F}\left(-C \partial_{\tau}^{2} E_{1}\right)=C \omega^{2} \hat{E}
$$

representing a gain that increases indefinitely for high frequencies. This is unphysical and therefore equation (52) becomes clearly unstable when $C>0$. Within the long-wave approximation, the large values of $\omega$ are disregarded. However, when we attempt to solve the asymptotic dynamical equation (52), all real frequencies are reintroduced de facto. Due to the instability, the high frequencies become large, which is inconsistent with the scaling. That is the origin of this unphysical behaviour. In order to force the asymptotic model to remain in the long-wave regime, this high-frequency instability must be removed. This can be performed in an ad hoc way by introducing an additional regularizing term proportional to $\left(-\partial_{\tau}^{4} E_{1}\right)$. However, the introduction of this regularizing term in the dynamical equation (52) can also be fully justified from the multiscale perturbation analysis as follows.

First we estimate $w_{3}$ from equation (48). The $a$-component of equation (48) is

$$
\mathrm{i} \partial_{\tau} \rho_{2 a}=-E_{1}\left(\mu \rho_{2 t}^{*}-\mu^{*} \rho_{2 t}\right)-E_{2}\left(\mu \rho_{1 t}^{*}-\mu^{*} \rho_{1 t}\right)+\mathrm{i} \lambda_{3 a}-\mathrm{i} \gamma_{a} \rho_{3}^{a} .
$$

From equations (34) and the expression of $\rho_{2 t}$ we see that both $\rho_{1 t}$ and $\rho_{2 t}$ are $O\left(E_{j}\right.$ ) (we denote $O\left(E_{j}\right)=O\left(E_{1}\right) \cap O\left(E_{2}\right), O\left(E_{j}^{2}\right)=O\left(E_{1}^{2}\right) \cap O\left(E_{2}^{2}\right) \cap O\left(E_{1} E_{2}\right)$, and so on); hence, from equation (41) we get

$$
w_{3}=\frac{2 \lambda_{3}^{b}}{\gamma_{a}}+O\left(E_{j}^{2}\right)
$$

Equation $(S)$ at order $\varepsilon^{4}$ is then

$\mathrm{i} \partial_{\tau} \rho_{3}=\left[H_{0}, \rho_{4}\right]-E_{4}\left[\mu, \rho_{0}\right]-E_{3}\left[\mu, \rho_{1}\right]-E_{2}\left[\mu, \rho_{2}\right]-E_{1}\left[\mu, \rho_{3}\right]+\mathrm{i} \Lambda_{4}-\mathrm{i} R_{4}$.

The $t$-component of equation (62) is written as

$$
\mathrm{i} \partial_{\tau} \rho_{3 t}=-\Omega \rho_{4 t}-E_{4} \mu w_{0}-E_{3} \mu w_{1}-E_{3} \mu w_{2}-E_{1} \mu w_{3}-\mathrm{i} \gamma_{t} \rho_{4 t} .
$$

From the expression of $\rho_{3 t}$, we see that

$$
\rho_{3 t}=\frac{-2 \lambda_{2 b} \mu}{\gamma_{a}\left(\Omega+\mathrm{i} \gamma_{t}\right)} E_{1}+\frac{\mathrm{i} \mu w_{0}}{\left(\Omega+\mathrm{i} \gamma_{t}\right)^{2}} \partial_{\tau} E_{1}+\frac{\mu w_{0}}{\left(\Omega+\mathrm{i} \gamma_{t}\right)^{3}} \partial_{\tau}^{2} E_{1}+F\left(E_{j}, j \geqslant 2\right)+O\left(E_{j}^{3}\right),
$$

in which $F\left(E_{j}, j \geqslant 2\right)$ holds for any quantity which depends on the $E_{j}(j \geqslant 2)$ but not on $E_{1}$. Then by using equations (41) and (61) we find that

$$
\begin{array}{r}
\rho_{4 t}=\frac{-2 \lambda_{3 b} \mu}{\gamma_{a}\left(\Omega+\mathrm{i} \gamma_{t}\right)} E_{1}+\frac{\mathrm{i} 2 \lambda_{2 b} \mu}{\gamma_{a}\left(\Omega+\mathrm{i} \gamma_{t}\right)^{2}} \partial_{\tau} E_{1}+\frac{\mu w_{1}}{\left(\Omega+\mathrm{i} \gamma_{t}\right)^{3}} \partial_{\tau}^{2} E_{1} \\
-\frac{\mathrm{i} \mu w_{0}}{\left(\Omega+\mathrm{i} \gamma_{t}\right)^{4}} \partial_{\tau}^{3} E_{1}+F\left(E_{j}, j \geqslant 2\right)+O\left(E_{j}^{3}\right) .
\end{array}
$$

Hence

$$
P_{4}=a_{0} E_{1}+b_{0} \partial_{\tau} E_{1}+c_{0} \partial_{\tau}^{2} E_{1}+d_{0} \partial_{\tau}^{3} E_{1}+F\left(E_{j}, j \geqslant 2\right)+O\left(E_{j}^{3}\right)
$$

where the coefficients $a_{0}, b_{0}, c_{0}$ and $d_{0}$ can be easily computed, and analogously for $P_{4}^{\prime}$. In the evolution equation (51), the quantity $\left(E_{3}+P_{3}+P_{3}^{\prime}\right)$ is replaced with

$$
\left(E_{3}+P_{3}+P_{3}^{\prime}+\varepsilon P_{4}+\varepsilon P_{4}^{\prime}\right)
$$


and on the right-hand side of equation (52) appears a set of corrective terms of order $\varepsilon$. The terms which come from $F\left(E_{j}, j \geqslant 2\right)$ would be cancelled, at following order, by analogous terms on the left-hand side of equation (52); hence, they must be dropped. The nonlinear terms coming from $O\left(E_{j}^{3}\right)$ will be neglected, too. Regarding the terms linear in $E_{1}$, the coefficients $B, C, D$, should be replaced by

$$
\begin{aligned}
& B^{\prime}=B+\frac{\varepsilon V}{2}\left(c_{0}+c_{0}^{\prime}\right), \\
& C^{\prime}=C+\frac{\varepsilon V}{2}\left(b_{0}+b_{0}^{\prime}\right), \\
& D^{\prime}=D-\frac{\varepsilon V}{2}\left(a_{0}+a_{0}^{\prime}\right),
\end{aligned}
$$

respectively. However, these small corrections to the coefficients can be neglected. The last term $d_{0} \partial_{\tau}^{3} E_{1}$ in equation (66) (and the analogous 'prime' one) yields the following term:

$$
-\frac{\varepsilon V}{2}\left(d_{0}+d_{0}^{\prime}\right) \partial_{\tau}^{4} E_{1}
$$

to be added to the right-hand side of (52).

The quantity $d_{0}$ can be evaluated as

$$
d_{0}=\frac{2 \gamma_{t}\left(\gamma_{t}^{2}-3 \Omega^{2}\right)|\mu|^{2} w_{0}}{\left(\Omega^{2}+\gamma_{t}^{2}\right)^{3}},
$$

and analogously for $d_{0}^{\prime}$. Finally, equation (52) is replaced with the regularized one

$$
\partial_{\zeta} E_{1}=-A \partial_{\tau} E_{1}^{3}-B \partial_{\tau}^{3} E_{1}-C \partial_{\tau}^{2} E_{1}+D \partial_{\tau} E_{1}-G \partial_{\tau}^{4} E_{1}-\Gamma E_{1}
$$

where

$$
G=\frac{\varepsilon V}{2}\left(\frac{2 \gamma_{t}\left(\gamma_{t}^{2}-3 \Omega^{2}\right)|\mu|^{2} w_{0}}{\left(\Omega^{2}+\gamma_{t}^{2}\right)^{3}}+\frac{2 \gamma_{t}^{\prime}\left(\gamma_{t}^{\prime 2}-3 \Omega^{\prime 2}\right)\left|\mu^{\prime}\right|^{2} w_{0}^{\prime}}{\left(\Omega^{\prime 2}+\gamma_{t}^{\prime 2}\right)^{3}}\right)
$$

The partial differential evolution equation (73), which is a non-SVEA version of Lorenz-Haken laser equation, constitutes the central result of this work. It adequately describes the physics of few-optical-cycle dissipative solitons beyond the SVEA. The nonlinear term $-A \partial_{\tau} E_{1}^{3}$ accounts for the Kerr effect, while the term $-B \partial_{\tau}^{3} E_{1}$ accounts for dispersion. These terms and the corresponding coefficients $A$ and $B$ have the same expressions as in the conservative counterpart of equation (73), which is the mKdV equation derived in [13]. The term $D \partial_{\tau} E_{1}$ describes a change in the pulse (phase and group) velocity. Expression (56) of $D$ shows that this velocity change is due to a gain effect. The three other terms account for a frequency-dependent gain-loss: the main term is $-C \partial_{\tau}^{2} E_{1}$, which accounts for a broadband gain. However, the width of the gain spectrum must be finite, which is ensured by the term $-G \partial_{\tau}^{4} E_{1}$. On the other hand, linear losses must be introduced to avoid excessive amplification they are accounted for by the term $-\Gamma E_{1}$. It is thus seen that equation (73) contains all terms involved by a one-dimensional description of a laser setup, such as nonlinearity, dispersion and frequencydependent gain and losses, except one term: the nonlinear gain, or effective saturable absorber. We will see below that this term is not necessary for stabilization of the FCP.

\section{Numerical simulations}

The dynamical equation (73) is solved numerically by means of an exponential time differencing scheme, of second-order Runge-Kutta type [55] and we implement absorbing 


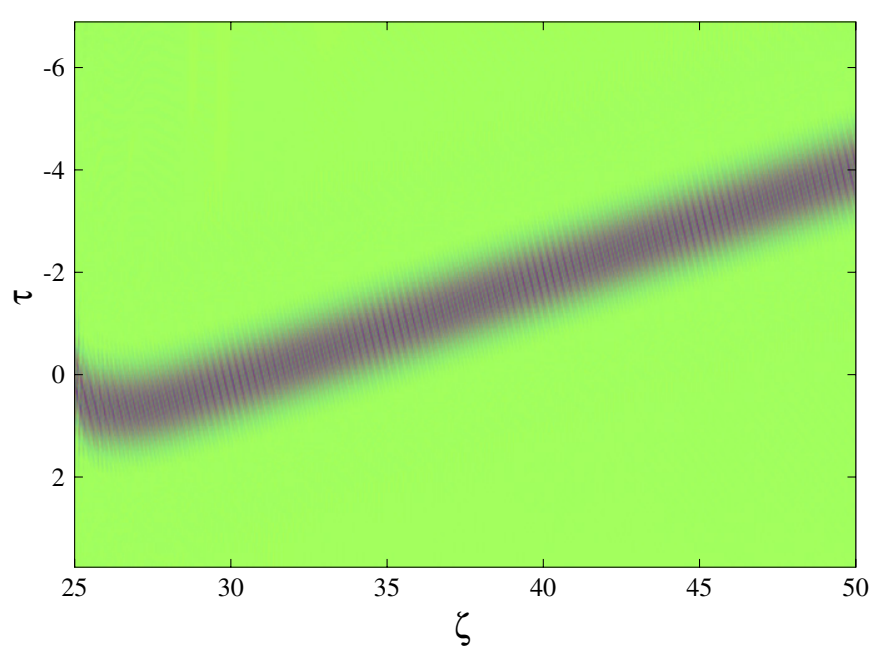

Figure 1. Evolution of a dissipative FCP soliton.

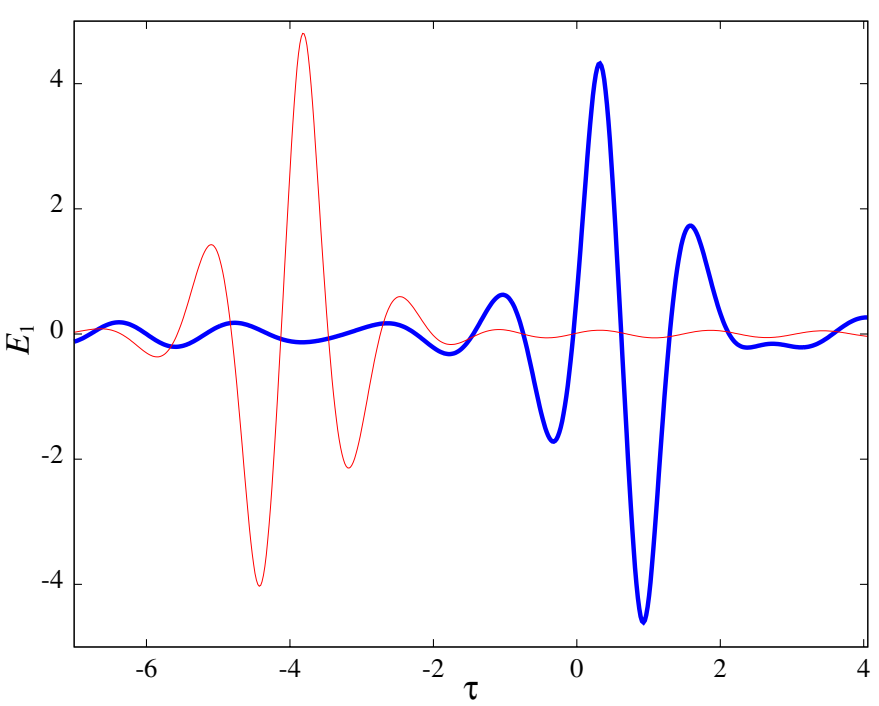

Figure 2. Evolution of a dissipative FCP soliton. Blue thick line: $z=26$; red thin line: $z=50$.

boundary conditions in the numerical simulations. We take the following numerical values of the parameters: the nonlinear coefficient $A=1$ (i.e. we consider a focusing optical medium), the third-order dispersion coefficient $B=1, C=0.1$, which correspond to gain, $G=0.002$ (corresponding to losses), $D=-48.836$ (a velocity adjusted in such a way that the soliton remains in the computation box) and $\Gamma=1$ (corresponding to losses). For this specific set of parameters we obtain the stable few-optical-cycle dissipative soliton shown in figures 1 and 2. The initial field was a breather solution of the underlying $\mathrm{mKdV}$ equation. The pulse was already propagated from $z=0$ to $z=25$ before the beginning of the numerical simulation; however, there is still some reshaping of the pulse, because the velocity $D$ of the frame has been readjusted just at $z=25$. Note that due to finite width of the numerical box, the dissipative soliton is not exactly invariant with respect to a change in the value of $D$. The 

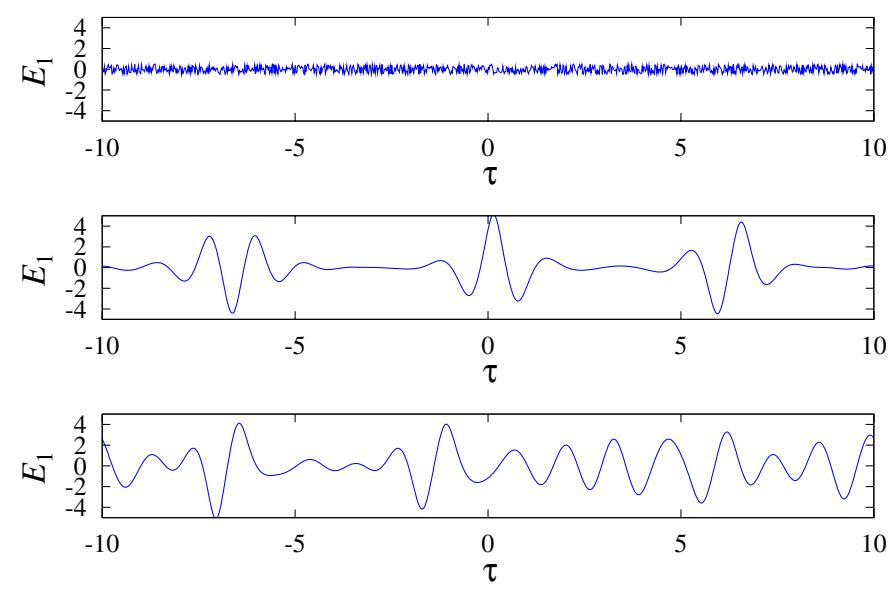

Figure 3. Evolution of the electric field from noise. (a) The input $(z=0)$. (b) Three dissipative FCP solitons are formed $(z=31.9)(c)$ The interaction of many FCP solitons appears as chaotic $(z=75)$

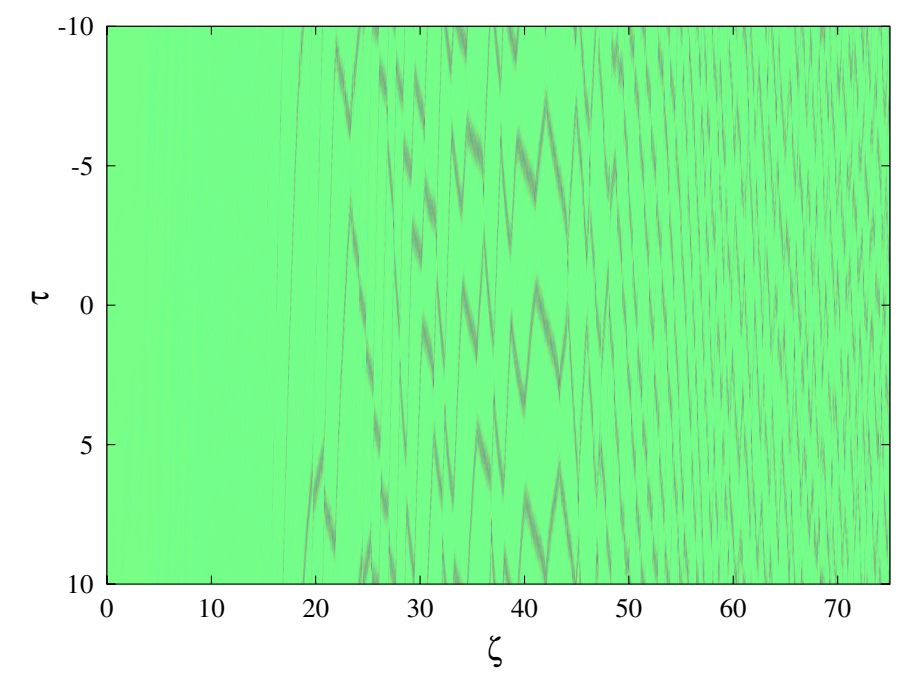

Figure 4. Evolution of the electric field from noise (same as in figure 3, but in the $(z, t)$ plane).

above FCP propagates thus in a stable way. Its stabilization does not require any effective saturable absorber, or nonlinear gain term in equation (73).

The above computation uses initial data close to the final FCP, hence describes the stability of the pulse rather in the medium used as an amplifier than really in the laser cavity. In order to study the latter issue, we use a random input and see whether a self-starting behaviour can be observed. Using periodic boundary conditions, it is found that FCPs form spontaneously (figures 3(b), and figure 4, left-hand side); however, their number in the numerical box increases during propagation, and they interact directly or by means of other components having a different velocity (figure 4). The result of the interaction and superposition of many FCPs appears as irregular oscillations, with an apparently chaotic behaviour (figure 3(c), and figure 4, right-hand side). It is incoherent light, and in this sense incoherent light can be seen 


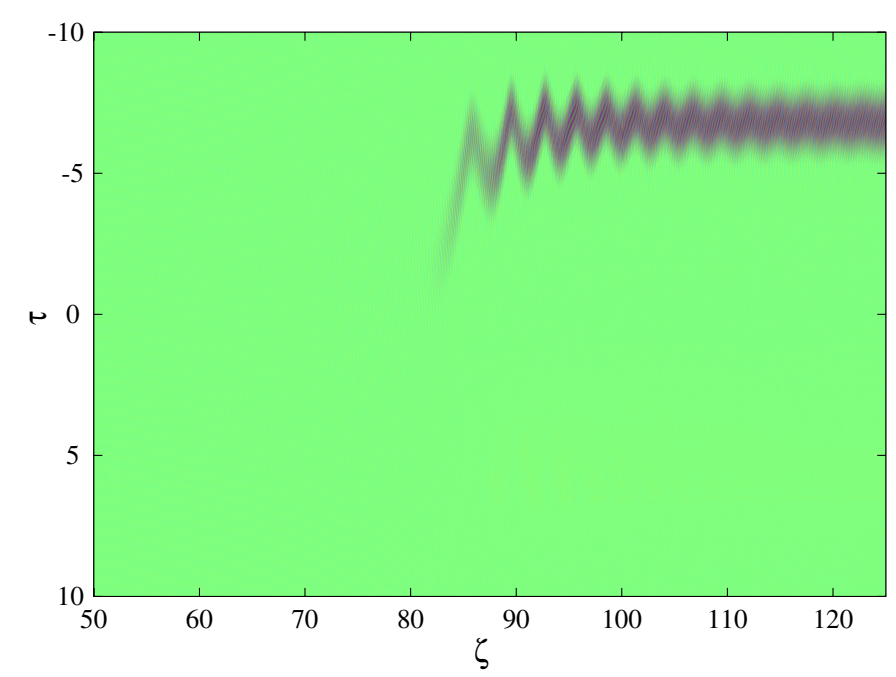

Figure 5. Formation of a FCP soliton from noise in the presence of velocity filtering yielded by absorbing boundary conditions.

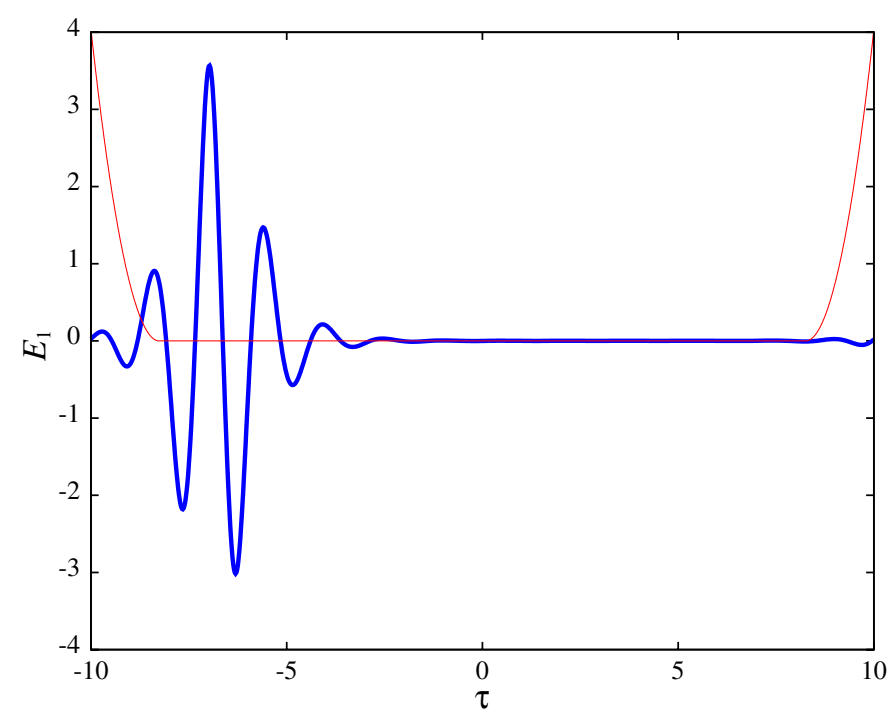

Figure 6. The FCP soliton formed from noise shown in figure 5 (thick blue line). The thin red line shows the transverse variations of the linear absorption coefficient $\alpha$ producing the absorbing boundary conditions (more exactly $\alpha / 15$ ).

as a superposition of FCPs. Thus, in order to produce a FCP laser, the question is not, how a FCP can be produced, but how it can be isolated. In the frame of the numerical computation, the absorbing boundary conditions achieve it (figure 5). However, the obtained FCP soliton is locked to the edge of the pumped zone (figure 6). Hence the mechanism of stabilization is closely related to the temporal localization of pumping realized this way. It strongly differs from the situation of the figures 1 and 2, in which initial data close to the final FCP were set. In this case, the absorbing boundary condition only ensured that the pulse did not be submitted to large perturbation coming from the outside. It only proved pulse stability for 
the cavity working as an amplifier. The situation changes radically when the FCP is expected to start from noise, since the perturbations also can self-start from noise. It may be thought that an effective saturable absorber could stabilize the formation of an isolated pulse. We run many calculations using an additional nonlinear gain/loss term in equation (73), in the (ad hoc) form $-A^{\prime} E_{1}^{3},-A^{\prime} \partial_{\tau}^{2}\left(E_{1}^{3}\right)$ or $-A^{\prime}\left(E_{1}^{2}\right) \partial_{\tau}^{2} E_{1}$, with various values of the coefficient $A^{\prime}$. The purely cubic term does not modify significantly the stability properties of the pulse, while the other forms, which are frequency dependent, destabilize it completely, leading either to a continuous wave or to numerical collapse depending on the sign of $A^{\prime}$.

The absorbing boundary conditions are thus the only way to stabilize the pulse. The precise identification of the physical mechanism they are modelling requires further investigation. Indeed, careful observation of figure 4 shows that they act as a velocity-dependent filter. It must be noted that a temporally localized pumping was also used in the numerical simulations of [49]. In the strict sense, the numerical result shows that a single FCP may be formed if the pumping is temporally localized, which can be achieved by pumping with a short pulse (but not a FCP). On the other hand, velocity filtering is not an exotic process. It has been used to manage the structure of soliton trains; the suppression of the highest velocities yields a 'cooling' which transforms a train with a 'gas' structure into one with a 'glass', and then 'crystal' structure [56]. Further, recall that gain saturation is not taken into account in our analysis. In the frame of SVEA, the gain saturation is known to limit the number of pulses [57], and it has been shown that it could stabilize mode locking in solid state lasers [58]. The accounting of gain saturation in the model is left for further study. A third interpretation could be sought in the frame of KLM, since it yields a temporal response in terms of amplitudedependent gain/loss; it is not excluded that KLM would produce the required velocity filtering. However, full resolution of the spatio-temporal counterpart to equation (73) is necessary to study this eventuality. It is thus left for further study. The aim of the present paper was to derive a non-SVEA model of a laser cavity, and to show that such an approach could describe dissipative FCP soliton formation. It is restricted to one dimension as a first attempt in this direction.

\section{Conclusion}

In conclusion, we have introduced a model based on the Maxwell-Bloch equations for an ensemble of $(2 \times 2)$-level atoms, which takes into account both the losses due to the optical field reflection at the laser cavity mirrors and the ones occurring in the optical medium itself. We have used the multiscale approach up to the third order in a certain small perturbation parameter. As a result of this powerful reductive perturbation method, a generalized mKdV equation containing gain and loss terms has been derived. Numerical simulations have clearly proved its suitability for describing the physics of few-optical-cycle dissipative solitons. A remarkable feature is that no nonlinear gain/loss term accounting for some effective saturable absorber is required. However, a single dissipative FCP soliton is formed only if some velocity filtering or temporal localization of pumping is introduced. The study of the mechanism allowing to realize such effect is left for further investigation. The results obtained in this work can also be generalized to $(2+1)$-dimensional few-cycle-pulse propagation models for dissipative optical solitons.

\section{Acknowledgments}

This work was supported in part by the Romanian Ministry of Education and Research (Grant No. IDEI-497/2009). 


\section{References}

[1] Brabec T and Krausz F 2000 Intense few-cycle laser fields: frontiers of nonlinear optics Rev. Mod. Phys. 72 545-91

[2] Wegener M 2005 Extreme Nonlinear Optics (Berlin: Springer)

[3] Scrinzi A, Ivanov M Yu, Kienberger R and Villeneuve D M 2006 Attosecond physics J. Phys. B: At. Mol. Opt. Phys. 39 R1-37

[4] Krausz F and Ivanov M 2009 Attosecond physics Rev. Mod. Phys. 81 163-234

[5] Tan X, Fan X, Yang Y and Tong D 2008 Time evolution of few-cycle pulse in a dense V-type three-level medium J. Mod. Opt. 55 2439-48

[6] Rosanov N N, Semenov V E and Vyssotina N V 2007 Collisions of few-cycle dissipative solitons in active nonlinear fibers Laser Phys. 17 1311-6

[7] Rosanov N N, Semenov V E and Vysotina N V 2008 Few-cycle dissipative solitons in active nonlinear optical fibres Quantum Electron. 38 137-43

[8] Nazarkin A 2006 Nonlinear optics of intense attosecond light pulses Phys. Rev. Lett. 97163904

[9] Brabec T and Krausz F 1997 Nonlinear optical pulse propagation in the single-cycle regime Phys. Rev. Lett. 78 3282-5

[10] Tognetti M V and Crespo H M 2007 Sub-two-cycle soliton-effect pulse compression at 800 nm in photonic crystal fibers J. Opt. Soc. Am. B 24 1410-5

[11] Voronin A A and Zheltikov A M 2008 Soliton-number analysis of soliton-effect pulse compression to singlecycle pulse widths Phys. Rev. A 78063834

[12] Mel'nikov I V, Mihalache D, Moldoveanu F and Panoiu N-C 1997 Quasiadiabatic following of femtosecond optical pulses in a weakly excited semiconductor Phys. Rev. A 56 1569-76

[13] Leblond H and Sanchez F 2003 Models for optical solitons in the two-cycle regime Phys. Rev. A 67013804

[14] Mel'nikov I V, Leblond H, Sanchez F and Mihalache D 2004 Nonlinear optics of a few-cycle optical pulse: slow-envelope approximation revisited IEEE J. Sel. Top. Quantum Electron. 10 870-5

[15] Leblond H, Sazonov S V, Mel'nikov I V, Mihalache D and Sanchez F 2006 Few-cycle nonlinear optics of multicomponent media Phys. Rev. A 74063815

[16] Leblond H, Mel'nikov I V and Mihalache D 2008 Interaction of few-optical-cycle solitons Phys. Rev. A 78043802

[17] Dodd R K, Eilbeck J C, Gibbon J D and Morris H C 1982 Solitons and Nonlinear Wave Equations (London: Academic)

[18] Ablowitz M J and Segur H 1981 Solitons and the Inverse Scattering Transform (Philadelphia: SIAM)

[19] Konno K, Kameyama W and Sanuki H 1974 Effect of weak dislocation potential on nonlinear wave propagation in anharmonic crystal J. Phys. Soc. Japan 37 171-6

[20] Mel'nikov I V, Mihalache D and Panoiu N-C 2000 Localized multidimensional femtosecond optical pulses in an off-resonance two-level medium Opt. Commun. 181 345-51

[21] Leblond H, Sanchez F, Mel'nikov I V and Mihalache D 2005 Optical solitons in a few-cycle regime: Breakdown of slow-envelope approximation Math. Comput. Simul. 69 378-88

[22] Sazonov S V 2001 Extremely short and quasi-monochromatic electromagnetic solitons in a two-component medium J. Exp. Theor. Phys. 92 361-73

[23] Bugay A N and Sazonov S V 2004 Faster-than-light propagation of electromagnetic solitons in nonequilibrium medium taking account of diffraction J. Opt. B: Quantum Semiclass. Opt. 6 328-35

[24] Schäfer T and Wayne C E 2004 Propagation of ultra-short optical pulses in cubic nonlinear media Physica D 196 90-105

[25] Chung Y, Jones C K R T, Schäfer T and Wayne C E 2005 Ultra-short pulses in linear and nonlinear media Nonlinearity 18 1351-74

[26] Sakovich A and Sakovich S 2005 The short pulse equation is integrable J. Phys. Soc. Japan $74239-41$

[27] Sakovich A and Sakovich S 2006 Solitary wave solutions of the short pulse equation J. Phys. A: Math. Gen. 39 L361-7

[28] Pietrzyk M, Kanattšikov I and Bandelow U 2008 On the propagation of vector ultra-short pulses J. Nonlinear Math. Phys. 15162

[29] Amiranashvili Sh, Vladimirov A G and Bandelow U 2008 Solitary-wave solutions for few-cycle optical pulses Phys. Rev. A 77063821

[30] Kim A V, Skobelev S A, Anderson D, Hansson T and Lisak M 2008 Extreme nonlinear optics in a Kerr medium: Exact soliton solutions for a few cycles Phys. Rev. A 77043823

[31] Kozlov S A and Sazonov S V 1997 Nonlinear propagation of optical pulses of a few oscillations duration in dielectric media J. Exp. Theor. Phys. 84 221-8 
[32] Bespalov V G, Kozlov S A, Shpolyanskiy Yu A and Walmsley I A 2002 Simplified field wave equations for the nonlinear propagation of extremely short light pulses Phys. Rev. A 66013811

[33] Berkovsky A N, Kozlov S A and Shpolyanskiy Yu A 2005 Self-focusing of few-cycle light pulses in dielectric media Phys. Rev. A 72043821

[34] Skobelev S A, Kartashov D V and Kim A V 2007 Few-optical-cycle solitons and pulse self-compression in a Kerr medium Phys. Rev. Lett. 99203902

[35] Leblond H and Mihalache D 2009 Few-optical-cycle solitons: modified Korteweg-de Vries-sine Gordon equation versus other non-slowly varying envelope approximation models Phys. Rev. A 79063835

[36] Haus H A, Fujimoto J G and Ippen E P 1991 Structures for additive pulse mode locking J. Opt. Soc. Am. B 8 2068-76

[37] Aranson I S and Kramer L 2002 The world of the complex Ginzburg-Landau equation Rev. Mod. Phys. 74 99-143

[38] Akhmediev N N and Ankiewicz A 1997 Solitons: Nonlinear Pulses and Beams (London: Chapman and Hall)

[39] Leblond H, Salhi M, Hideur A, Chartier T, Brunel M and Sanchez F 2002 Experimental and theoretical study of the passively mode locked ytterbium-doped double-clad fiber laser Phys. Rev. A $\mathbf{6 5} 063811$

[40] Salhi M, Haboucha A, Leblond H and Sanchez F 2008 Theoretical study of figure-eight all fiber laser Phys. Rev. A 77033828

[41] Komarov A, Leblond H and Sanchez F 2005 Quintic complex Ginzburg-Landau model for ring fiber lasers Phys. Rev. E 72025604

[42] Grelu Ph, Béal J and Soto-Crespo J M 2003 Soliton pairs in a fiber laser: from anomalous to normal average dispersion regime Opt. Express 112238

[43] Lega J, Moloney J V and Newell A C 1994 Swift-Hohenberg equation for lasers Phys. Rev. Lett. 732978

[44] Haus H A 1975 Theory of mode locking with a slow saturable absorber IEEE J. Quantum Electron. QE-11 736

[45] Haus H A, Fujimoto J G and Ippen E P 1992 Analytic theory of additive pulse and Kerr lens mode locking IEEE J. Quantum Electron. 282086

[46] Brabec T, Spielmann Ch, Curley P F and Krausz F 1992 Kerr lens mode locking Opt. Lett. 171292

[47] Huang D, Ulman M, Acioli L H, Haus H A and Fujimoto J G 1992 Self-focusing-induced saturable loss for laser mode locking Opt. Lett. 17511

[48] Herrmann J 1994 Theory of Kerr-lens mode locking: role of self-focusing and radially varying gain J. Opt. Soc. Am. B 11498

[49] Jirauschek C, Kärtner F X and Morgner U 2003 Spatiotemporal Gaussian pulse dynamics in Kerr-lens mode locked lasers J. Opt. Soc. Am. B 201356

[50] Ell R et al 2001 Generation of 5-fs pulses and octave-spanning spectra directly from a Ti:sapphire laser Opt. Lett. 26373

[51] Leblond H 2008 The reductive perturbation method and some of its applications J. Phys. B: At. Mol. Opt. Phys. 41043001

[52] Lorenz E N 1963 Deterministic aperiodic flow J. Atmos. Sci. 20 130-41

[53] Haken H 1975 Analogy between higher instabilities in fluids and lasers Phys. Lett. A 53 77-8

[54] Komarov A, Leblond H and Sanchez F 2005 Quintic complex Ginzburg-Landau model for ring fiber lasers Phys. Rev. E 72025604

[55] Cox S M and Matthews P C 2002 Exponential time differencing for stiff Systems J. Comput. Phys. 176 430-55

[56] Rutz S and Mitschke F 2000 Towards thermodynamics of solitons: cooling J. Opt. B: Quantum Semiclass. Opt. 2364

[57] Haboucha A, Leblond H, Salhi M, Komarov A and Sanchez F 2008 Analysis of soliton pattern formation in passively mode locked fiber lasers Phys. Rev. A 78043806

[58] Piché M and Salin F 1993 Self-mode locking of solid-state lasers without apertures Opt. Lett. 181041 